\title{
COMPUTING CONFORMAL MAPS OF FINITELY CONNECTED DOMAINS ONTO CANONICAL SLIT DOMAINS
}

\author{
VALENTIN V. ANDREEV AND TIMOTHY H. MCNICHOLL
}

\begin{abstract}
We continue the research initiated in [2] on the computability of conformal mapping of multiply connected domains by showing that the conformal maps of a finitely connected domain onto the canonical slit domains can be computed uniformly from the domain and its boundary. Along the way, we demonstrate the computability of finding analytic extensions of harmonic functions and solutions to Neuman problems. These results on conformal mapping then follow easily from M. Schiffer's constructions [17].
\end{abstract}

\section{INTRODUCTION}

Our goal here is to continue the work initiated in [2] on the computability of conformal mapping of finitely connected domains. We begin with an explanation of and motivation for the problems we are to consider.

Let $\hat{\mathbb{C}}$ denote the extended complex plane. A domain is an open connected subset of $\hat{\mathbb{C}}$. A domain is degenerate if a component of its complement consists of a single point. A domain is $n$-connected if its complement has precisely $n$ connected components and finitely connected if it is $n$-connected for some $n$.

When studying conformal mappings between domains in the extended complex plane, it is convenient for both theoretical and practical purposes to introduce the so-called canonical domains and to study conformal maps of arbitrary domains onto these canonical domains. If the domain is 1-connected and non-degenerate, the canonical domain is the unit disk, $\mathbb{D}$. There is now an abundance of literature on constructive, computable, and computational conformal mapping of 1-connected domains. For example, a constructive proof of the Riemann Mapping Theorem appears in [3]. In [11], P. Hertling proved an effective Riemann Mapping Theorem which, roughly speaking, states that there is a Turing machine which given an enumeration of all closed rational rectangles contained in a non-degenerate 1-connected domain $D$, and a list of all open rational rectangles which intersect the boundary of $D$, computes a conformal map $f$ of $D$ onto $\mathbb{D}$ in the sense that it enumerates all pairs of the form $\left(R_{1}, R_{2}\right)$ where $R_{1}, R_{2}$ are open rational rectangles such that $\overline{R_{1}} \subseteq D$ and $f\left[\overline{R_{1}}\right] \subseteq R_{2}$. Here, a rational rectangle is a rectangle with the property that each coördinate of any one of its vertices is a rational number.

In the case of doubly connected non-degenerate domains, the canonical domain is the annulus $\left\{z \in \mathbb{C}: r_{1}<|z|<r_{2}\right\}$. The modulus of this annulus is $r_{2} / r_{1}$.

2000 Mathematics Subject Classification. 03F60, 30C20, 30C30, and 30C85.

Authors' note: It is our understanding that R. Rettinger has obtained some of these results by different methods.

The results of this paper were presented at the Sixth International Conference on Computability and Complexity in Analysis, Ljubljana, Slovenia, 2009. A preliminary version appeared in the proceedings of this conference. 
It is well-known that annuli with different moduli are not conformally equivalent (see, e.g., [15] p. 333). When considering conformal mappings of domains with connectivity $n \geq 3$, it is convenient to consider canonical domains with different geometric characteristics. In their seminal book, M. Pour-El and I. Richards posed the problem of determining the computability of conformal mapping of finitely connected domains onto various canonical domains [16]. This is a problem which, in a different guise, has also been the subject of much research in classical complex analysis. See, for example, [10] and [17]. The latter will be the starting point for much of our investigations in this paper.

Paul Koebe [12] outlined an iteration method for finding the conformal mapping from a non-degenerate $n$-connected domain to a circular domain (i.e. a domain whose complement consists of $n$ disjoint closed disks). The convergence of his method was proved by Gaier [7]. However, this proof is based on a priori knowledge of the target circular domain. The computability of conformal mapping onto circular domains, in the sense of the aforementioned result of Hertling, was first proved by Andreev, Daniel, and McNicholl in [2]. In this proof, the Koebe construction was still used to generate approximations, but the error is estimated without prior knowledge of the target circular domain. The circular domains are the canonical domains in the recent constructions of the Schwarz-Christoffel mappings for domains that are sufficiently separated (see [5] and [6] and the refernces therein) and have been used as canonical domains in aircraft engineering as early as 1928 [1] and later by Halsey [9]. For numerous applications to nonlinear problems in mechanics see the monograph [14].

Paul Koebe [13] introduced several of the canonical slit domains. These are defined as follows.

The slit disk domain. Let $\mathbb{D}$ denote the unit disk centered at the origin. These domains are obtained by removing finitely many arcs from $\mathbb{D}$. Each of these arcs must be an arc of a circle centered at the origin.

The slit annulus. These domains are obtained by removing finitely many arcs from an annulus whose outer circle is $\partial \mathbb{D}$. Again, each of these arcs must be an arc of a circle centered at the origin.

The circular slit domain. These domains are obtained by removing from $\hat{\mathbb{C}}$ one or more arcs. Again, each of these arcs must be an arc of a circle centered at the origin.

The radial slit domain. These domains are obtained by removing from $\hat{\mathbb{C}}$ one or more line segments which do not pass through the origin. Each of these line segments, when extended indefinitely in both directions, must yield a line that passes through the origin.

The parallel slit domain. These domains are obtained by removing from $\hat{\mathbb{C}}$ one or more parallel line segments.

Here, we will prove that conformal maps onto domains in these five classes can be computed from a non-degenerate finitely connected domain $D$ and its boundary in the sense of the aforementioned result of Hertling on the Riemann Mapping Theorem. To do so, we will first demonstrate the computability of two important 
operations in harmonic function theory: analytic extension and solution of Neuman problems. As these operations are frequently used in classical function theory, we anticipate that these results will be have broad application in the future development of computable function theory. By using the famous constructions of $\mathrm{M}$. Schiffer [17], we then obtain as almost immediate consequences of these very widely applicable results, very simple proofs of the computability of conformal mapping onto the slit domains. In addition, Schiffer's constructions show that these maps can all be expressed in terms of Green's function. Hence, the complexity of these computations should not be very far removed from the complexity of computing the Green's function for a domain.

In order to state our results in the most uniform manner possible, we will use the Type-Two Effectivity approach to computable analysis based on naming systems and Turing machines which transform names of function arguments into names of the corresponding function values. See [18] for a thorough and detailed development. We will use the naming systems for $\widehat{\mathbb{C}}$ and its hyperspaces developed in [2]. These are based on the development in[19]. For the sake of completeness, we restate these here.

First of all, we use the basis for the standard topology on $\hat{\mathbb{C}}$ consisting of all open rational rectangles as well as all sets of the form $\hat{\mathbb{C}}-\bar{R}$ where $R$ is an open rational rectangle that contains the origin. A point in $\widehat{\mathbb{C}}$ is then named by an enumeration of all basic neighborhoods to which it belongs. We then name an open subset $U$ of $\hat{\mathbb{C}}$ by a list of all basic open sets whose closure is contained in $U$. A closed set is named by a list of all basic open sets which intersect it.

With regards to continuous functions into $\hat{\mathbb{C}}$, we wish to consider those whose domain is an open subset of $\hat{\mathbb{C}}$ and those whose domain is a closed subset of $\hat{\mathbb{C}}$. In the former case, a function $f$ is named by a list of all pairs of the form $\left(R_{1}, R_{2}\right)$ such that $R_{1}, R_{2}$ are open rational rectangles, $\overline{R_{1}} \subseteq \operatorname{dom}(f)$, and $f\left[\overline{R_{1}}\right] \subseteq R_{2}$. In the latter case, a name of such a function $f$ is a list of pairs of subbasic open sets $\left\{\left(U_{n}, V_{n}\right)\right\}_{n \in \mathbb{N}}$ that possesses the following two properties.

(1) Each $U_{n}$ contains at least one point of the domain of $f$ and its intersection with the domain of $f$ is mapped into $V_{n}$ by $f$.

(2) Whenever $x$ is in the domain of $f$ and $U, V$ are subbasic open sets that that $U$ contains $x$ and $V$ contains $f(x)$, there exists $n$ such that $x \in U_{n} \subseteq U$ and $V_{n} \subseteq V$.

The first property guarantees that each $\left(U_{n}, V_{n}\right)$ provides accurate partial information about $f$. The second ensures that we can obtain arbitrarily good estimates to any value of $f(x)$ from sufficiently good estimates to $x$. This naming system is equivalent to the $\delta_{2}$ naming system in Exercise 6.2.11 of [18].

Since these are the only naming systems we will use, we will suppress their mention. We will also talk about computations on objects when it is clear that we are really talking about computations with names of objects.

\section{BACKGROUND FROM COMPLEX ANALYSIS}

We wish to summarize here the definitions and results about harmonic and analytic functions which are pertinent to our investigations. We refer the reader who is interested in proofs to standard sources such as [4]. 
We begin with a few more definitions about domains. A boundary component of a domain is a connected component of its boundary. A domain is Jordan if its boundary components are Jordan curves. A Jordan domain is smooth if these curves are continuously differentiable.

A function $u$ on a domain $D$ is harmonic if

$$
\frac{\partial^{2} u}{\partial x^{2}}(z)+\frac{\partial^{2} u}{\partial y^{2}}(z)=0
$$

for all $z \in D$.

Suppose $u, v$ are harmonic functions on a domain $D$ and

$$
\begin{aligned}
& \frac{\partial u}{\partial x}=\frac{\partial v}{\partial y} \\
& \frac{\partial u}{\partial y}=-\frac{\partial v}{\partial x}
\end{aligned}
$$

Then, $v$ is said to be a harmonic conjugate of $u$. Equations (2.1) and (2.2) are known as the Cauchy-Riemann equations. It is well-known that $v$ is a harmonic conjugate of $u$ if and only if $u+i v$ is analytic.

Suppose $D$ is a Jordan domain and that $f$ is a bounded piecewise continuous function on its boundary. The resulting Dirichlet problem if to find a harmonic function $u$ on $D$ with the property that

$$
\lim _{z \rightarrow \zeta} u(z)=f(z)
$$

for all $\zeta \in \partial D$ at which $f$ is continuous. Solutions to Dirichlet problems always exist and are unique. The function $f$ is said to provide the boundary data for this problem. Now, for each $z \in D$, define $\omega\left(z, \Gamma_{j}, D\right)$ to be the value at $z$ of the solution to the Dirichlet problem with boundary data

$$
f(\zeta)=\left\{\begin{array}{cc}
1 & \zeta \in \Gamma_{j} \\
0 & \text { otherwise }
\end{array}\right.
$$

The function $\omega$ is called harmonic measure. In fact, the domain of $\omega$ in its second variable can be extended to all Borel subsets of $\partial D$. However, this is much more generality than we shall need here. The reader who desires this further development can find an outstanding treatment in [8].

The normal derivative of $u$ is denoted $\frac{\partial u}{\partial n}$ and is defined to be

$$
\left(\frac{\partial u}{\partial x} y^{\prime}(t)-\frac{\partial u}{\partial y} x^{\prime}(t)\right) \frac{1}{\left|x^{\prime}(t)+i y^{\prime}(t)\right|}
$$

when $(x, y)$ is a positively oriented smooth Jordan curve. In this case, we also define

$$
\frac{\partial u}{\partial s}=\left(\frac{\partial u}{\partial x} x^{\prime}(t)+\frac{\partial u}{\partial y} y^{\prime}(t)\right) \frac{1}{\left|x^{\prime}(t)+i y^{\prime}(t)\right|} .
$$

The Cauchy-Riemann equations can now be rewritten as

$$
\begin{aligned}
& \frac{\partial u}{\partial n}=\frac{\partial v}{\partial s} \\
& \frac{\partial v}{\partial n}=-\frac{\partial u}{\partial s} .
\end{aligned}
$$


If $u$ is harmonic, and if $\gamma$ is a boundary component of $\operatorname{dom}(u)$, then the period of the conjugate of $u$ about $\gamma$ is defined to be

$$
\frac{1}{2 \pi} \int_{\gamma_{0}} \frac{\partial u}{\partial n} d s
$$

where $d s={ }_{d f}\left|\gamma_{0}^{\prime}(t)\right| d t$ is the differential of arc length and $\gamma_{0} \subseteq \operatorname{dom}(u)$ is homotopic to $\gamma$.

Suppose $f$ is a conformal map of a domain $D$ onto a domain $D_{1}$, and that $\gamma, \gamma_{1}$ are boundary components of $D, D_{1}$ respectively. Suppose that whenever $\left\{z_{n}\right\}_{n=0}^{\infty}$ is a sequence of points in $D$ such that $\lim _{n \rightarrow \infty} d\left(z_{n}, \gamma\right)=0, \lim _{n \rightarrow \infty} d\left(f\left(z_{n}\right), \gamma_{1}\right)=0$. We say that $f$ maps $\gamma$ to $\gamma_{1}$.

We will follow the convention of identifying a curve with its parameterizations. However, a name of a curve is always a name of one of its parameterizations from which a name of the curve as a closed set can be computed.

\section{A summary of PREvious RESUlts}

The following are all proven in [2] and form the cornerstone for our work.

Theorem 3.1 (Computable differentiation of harmonic functions). From a name of a harmonic function, $u$, we may compute a name of $\left.u^{\prime}\right|_{\mathbb{C}}$.

Theorem 3.2 (Weak Computable Carathéodory Theorem). Given names of parameterizations of smooth arcs $\gamma_{1}, \ldots, \gamma_{n}$ and their derivatives, if $\gamma_{1}+\ldots+\gamma_{n}$ is a Jordan curve, then we can compute a conformal map of the interior of this curve onto $\mathbb{D}$ as well as the homeomorphic extension to the boundary. We can compute the inverse map on $\overline{\mathbb{D}}$ as well.

Theorem 3.3 (Computable Solution of Dirichlet Problems). Given a name of a Jordan domain $D$ and names of smooth $\gamma_{1}, \ldots, \gamma_{n}$ and their derivatives, if $\gamma_{1}, \ldots, \gamma_{n}$ are the distinct boundary components of $D$, and if we are also given a name of a continuous $f: \partial D \rightarrow \mathbb{R}$, then we can compute a solution of the corresponding Dirichlet problem. Furthermore, we can compute an extension of this solution to $\bar{D}$.

Theorem 3.4 (Computable Harmonic Extension). Given a name of a domain $D$, a name of a harmonic $u: \bar{D} \rightarrow \mathbb{R}$, and names of conformal $f_{1}, \ldots, f_{n}$ such that

- $f_{j}(\infty)=\infty$,

- $\overline{\mathbb{D}} \subseteq \operatorname{dom}\left(f_{j}\right)$,

- $\gamma_{j}={ }_{d f} f_{j}[\partial \mathbb{D}]$ is a boundary component of $D$ on which $u$ is zero, and

- $\gamma_{1}, \ldots, \gamma_{n}$ are distinct,

we can compute a neighborhood of $D \cup\left(\bigcup_{j} \gamma_{j}\right)$, $D^{\prime}$, and a harmonic extension of $\left.u\right|_{D}$ to $D^{\prime}$.

Theorem 3.5 (Computability of the Riemann Matrix). Given the same initial data as in Theorem 3.3, we can compute a name of the period of the harmonic conjugate of $\omega\left(\cdot, \Gamma_{i}, D\right)$ around $\Gamma_{j}$. 


\section{Computability of analytic extension}

Suppose $u$ is a harmonic function with domain $D$. If $D$ is 1-connected, then a harmonic conjugate of $u$ may be defined by the equation

$$
v(\zeta)=\int_{\zeta_{1}}^{\zeta} \frac{\partial u}{\partial n} d s
$$

Hence, $u+i v$ is analytic. If $D$ is multiply connected, then the right side of (4.1) may depend on the path of integration. In this case, $u$ is said to have a multivalued harmonic conjugate. Otherwise, $u$ is said to have a single-valued harmonic conjugate. It follows that if $D$ is contained in the interior of one of its boundary components then $u$ has a single-valued harmonic conjugate if and only if its period around every other boundary component is zero.

It is well-known that if $D$ is finitely connected and bounded by smooth Jordan curves, one of which contains $D$ in its interior, then one can add a unique linear combination of the harmonic measure functions of the boundary components of $D$ to $u$ and obtain a function with a single-valued harmonic conjugate. Our first goal is to show that this can be done effectively.

Lemma 4.1 (Computability of analytic extension). Given a name of a harmonic function $u$ defined on a finitely connected domain, $D$, and names of the boundary components of $D, \gamma_{1}, \ldots, \gamma_{n}$, we may compute $b_{1}, \ldots, b_{n-1}$ such that

$$
u+\sum_{j=1}^{n-1} b_{j} \omega\left(\cdot, \gamma_{j}, D\right)
$$

has a single-valued harmonic conjugate provided $\gamma_{1}, \ldots, \gamma_{n}$ are smooth Jordan curves, $D$ is contained in the interior of $\gamma_{n}$, and we are also given names of $\gamma_{1}^{\prime}, \ldots, \gamma_{n}^{\prime}$.

Proof. Let $R_{k, j}$ be the period of $\omega\left(\cdot, \gamma_{j}, D\right)$ about $\gamma_{k}$.

We first want to compute the period of the conjugate of $u$ about each $\gamma_{k}$. Denote this period by $p_{k}$. To compute $p_{k}$, we want $\gamma_{k}$ to be positively oriented. This can be checked by using the winding number

$$
\int_{\gamma_{k}} \frac{1}{z-\zeta}
$$

We can effectively search for a rational rectangle $R$ such that $\bar{R} \subseteq \mathbb{C}-\gamma_{k}$ on which this winding number is non-zero. If this value is positive, we can in addition discover a positive lower bound on it. If it is negative, then we can in addition discover a negative upper bound on it. In the former case, we know $\gamma_{k}$ is positively oriented. Otherwise, it is negatively oriented in which case we can reparameterize it positively. Hence, we will assume without loss of generality that each $\gamma_{k}$ is positively oriented.

Now, let $R_{k, j}$ be the period of $\omega\left(\cdot, \gamma_{j}, D\right)$ about $\gamma_{k}$. It is well-known that the matrix $\left(R_{k, j}\right)_{k, j=1, \ldots, n-1}$ is invertible. (See, e.g., Section I.10 of [15].) To ensure that the function in (4.2) has no conjugate period about $\gamma_{k}, k=1, \ldots, n-1$, it suffices to show that

$$
R_{k, 1} b_{1}+\ldots+R_{k, n-1} b_{n-1}=-p_{k} .
$$

It now follows from the results in $[20]$ that $b_{1}, \ldots, b_{n-1}$ can be computed from the given information. 


\section{Computing solutions to the Neuman problem}

Let $D$ be a bounded domain with smooth boundary curves $\Gamma_{1}, \ldots, \Gamma_{n}$. Let $f \in C(\partial D)$, and suppose $\int_{\partial D} f d s=0$. The resulting Neuman problem is to find a harmonic function $u$ on $D$ such that

$$
\begin{aligned}
\frac{\partial u}{\partial n} & =f \text { on } \partial D \\
\int_{\partial D} u d s & =0
\end{aligned}
$$

Such solutions exist (see, e.g. Appendix B of [8]). Condition (5.2) ensures they are unique. Their computability will now be demonstrated by a well-known procedure (see e.g. proof of Theorem B.1 in [8]).

Theorem 5.1 (Computing solutions of Neuman problems). Given a name of a bounded domain $D$, names of $n$ smooth Jordan curves $\Gamma_{1}, \ldots, \Gamma_{n}$ which form its boundary components as well as names of their derivatives, and a name of $f \in C(\partial D)$ such that $\int_{\partial D} f d s=0$, one can compute a name of the solution of the resulting Neuman problem.

Proof. There is already a well-known 'procedure' for finding solutions to Neuman problems. The purpose of this proof is to explain this procedure and show that it can be implemented on a digital computing device.

By using the winding number and a simple search procedure, we can determine which of $\Gamma_{1}, \ldots, \Gamma_{n}$ contains $D$ in its interior. Without loss of generality, suppose $\Gamma_{n}$ is this curve.

As in the proof of Lemma 4.1, we can assume $\Gamma_{1}, \ldots, \Gamma_{n}$ are positively oriented.

Let $R_{j, k}$ be the period of the conjugate of $\omega_{k}$ about $\Gamma_{j}$. As noted in the proof of Lemma 4.1 , the matrix $\left(R_{j, k}\right)_{j, k=1, \ldots, n-1}$ is invertible. So, we can now compute the solution to the system of linear equations

$$
R_{j, 1} b_{1}+\ldots+R_{j, n-1} b_{n-1}=\int_{\Gamma_{j}} f d s j=1, \ldots, n-1 .
$$

Let

$$
f_{1}=f-\sum_{k=1}^{n-1} b_{k} \frac{\partial \omega_{k}}{\partial n} .
$$

It follows that $\int_{\Gamma_{j}} f_{1} d s=0$ if $j \in\{1, \ldots, n-1\}$.

It is an easy consequence of Green's Theorem that $R_{j, k}=R_{k, j}$. (See, e.g., Section I.10 of [15].) It is also easy to show that for each $j$, the sum of the periods of the harmonic conjugates of $\omega_{1}, \ldots, \omega_{n}$ is 0 . (One first notes that the sum of the harmonic measure functions is identically 1 on $\partial D$.) Since $\int_{\partial D} f d s=0$, it now follows by a fairly straightforward calculation that $\int_{\Gamma_{n}} f_{1} d s=0$.

We now wish to define a function $g$ on $\partial D$. We do so by defining it on each boundary component of $D$. When $\zeta \in \Gamma_{j}$, we let

$$
g(\zeta)=\int_{0}^{t_{0}} f_{1}\left(\Gamma_{j}(t)\right)\left|\Gamma_{j}^{\prime}(t)\right| d t
$$

where $t_{0}$ is such that $\Gamma_{j}\left(t_{0}\right)=\zeta$. Since $\int_{\Gamma_{j}} f_{1} d s=0$, it follows that the choice of $t_{0}$ is irrelevant when $\zeta=\Gamma_{j}(0)$. Hence, $g$ is well-defined.

It is now necessary to prove the following Lemma. 
Lemma 5.2. $g$ can be computed from the given data.

Proof. Let $\omega_{j}=\omega\left(\cdot, \Gamma_{j}, D\right)$.

Suppose we are given a name of a point $\zeta \in \partial D$ as input. From our name for a parametrization of $\Gamma_{j}$, we can compute names of $\Gamma_{j}$ as a closed subset of the plane as well as a name of the open set $\mathbb{C}-\Gamma_{j}$. (See, e.g., Theorem 6.2.4.4 of [18].) We then scan these names and our name for $\zeta$ until we find a rational rectangle $R$ and an index $j$ such that $\zeta \in R, R \cap \Gamma_{j} \neq \emptyset$, and $\bar{R} \cap \Gamma_{k}=\emptyset$ when $k \neq j$. Hence, we now know $\zeta \in \Gamma_{j}$. Begin computing a name for the function $h$ defined by

$$
h(t)=\int_{0}^{t} f_{1}\left(\Gamma_{j}(t)\right)\left|\Gamma_{j}^{\prime}(t)\right| d t .
$$

We now continue scanning our name for $\zeta$ and our generated names for $\Gamma_{j}(0)$ and $h$. Suppose that at some point in this process we discover disjoint rational rectangles $R_{1}, R_{2}$ such that $\zeta \in R_{1}$ and $\Gamma_{j}(0) \in R_{2}$. So, we now know $\zeta \neq \Gamma_{j}(0)$. Hence, there is exactly one value of $t$ for which $\Gamma_{j}(t)=\zeta$ and we can compute this value. (See, e.g. Corollary 6.3 .5 of [18].) Hence, we can now compute $g(\zeta)$ directly from the definition of $g$.

Suppose on the other hand that at some point in this process no such rational rectangles have been discovered. We then search the portions of these names read so far for $R,\left[a_{1}, b_{1}\right], \ldots,\left[a_{m}, b_{m}\right], R_{1}, \ldots, R_{m}, R^{\prime}, I_{1}$, and $I_{2}$ such that

(1) $\zeta, \Gamma_{j}(0) \in R$,

(2) $\Gamma_{j}$ maps $\left[a_{l}, b_{l}\right]$ into $R_{l}$,

(3) $[a, b]={ }_{d f} \bigcup_{l}\left[a_{l}, b_{1}\right]$ is a subinterval of $(0,1)$,

(4) $R_{l} \cap \bar{R}=\emptyset$,

(5) $h$ maps $[0, a]$ into $I_{1}$, and

(6) $h$ maps $[b, 1]$ into $I_{2}$.

If this search fails, then we continue scanning. If it succeeds, then, although we do not know yet if $\zeta=\Gamma_{j}(0)$, we do know that any $\Gamma_{j}$ preimage of $\zeta$ lies in $[0, a] \cup[b, 1]$. Note that $0 \in I_{1} \cap I_{2}$. So, we can list, for each successful search of this kind, $I_{1} \cup I_{2}$ as an interval that contains $g(\zeta)$. We can also in the future interleave listing of all rational intervals that contain $I_{1} \cup I_{2}$.

We now show that this process generates a name for $g(\zeta)$. Every interval listed contains $g(\zeta)$. So, we only need to show that every interval that contains $g(\zeta)$ is eventually listed. This is clearly true if $\zeta \neq \Gamma_{j}(0)$. Suppose $\zeta=\Gamma_{j}(0)$. It follows that there will be infinitely many successful search of the kind described above. It also follows that larger portions of these names are read, the diameter of $I_{1} \cup I_{2}$ will tend to 0 . It follows that a name of $0=g\left(\Gamma_{j}(0)\right)$ is written on the output tape.

We now compute the solution to the Dirichlet problem for $D$ with boundary data $g$. Call this solution $v_{1}$. We now compute $a_{1}, \ldots, a_{n-1}$ such that

$$
v={ }_{d f} v_{1}+\sum_{j=1}^{n-1} a_{j} \omega_{j}
$$

has a single-valued harmonic conjugate. Since $\omega_{j}$ is constant on each curve of $\partial D$,

$$
0=\int_{\zeta_{0}}^{\zeta_{1}} \frac{\partial \omega_{j}}{\partial s} d s
$$


whenever $\zeta_{0}, \zeta_{1} \in \Gamma_{k}$ for some $k$. It then follows that $\frac{\partial \omega_{j}}{\partial s}$ is 0 on each boundary curve, and so $\frac{\partial v}{\partial s}=f_{1}$ on $\partial D$. Compute $\xi \in D$. Let:

$$
\begin{aligned}
u_{1}\left(z_{0}\right) & =\int_{\xi}^{z_{0}} \frac{\partial(-v)}{\partial n} d s \\
u_{2} & =u_{1}+\sum_{j=1}^{n-1} b_{j} \omega_{j}
\end{aligned}
$$

Since $u_{1}$ is a harmonic conjugate of $-v$, it follows that the normal derivative of $u_{1}$ on $\partial D$ is $f_{1}$. It now follows that $f$ is the normal derivative of $u_{2}$ on $\partial D$. We now complete our computation by setting

$$
u=u_{2}-\int_{\Gamma} u_{2} d s
$$

If $D$ is a bounded domain bounded by smooth Jordan curves $\Gamma_{1}, \ldots, \Gamma_{n}$, then the Neuman function of $D, N_{D}$, is defined by the following conditions.

(1) $z \mapsto N(z, \zeta)+\log |z-\zeta|$ is harmonic.

(2) $\frac{\partial}{\partial n_{z}} N(z, \zeta)=-\frac{2 \pi}{L}$ on $\partial D$ where $L$ is the length of $\partial D$.

(3) $\int_{\partial D} N(z, \zeta) d s_{z}=0$.

Corollary 5.3. From names of $D, \Gamma_{1}, \ldots, \Gamma_{n}, \Gamma_{1}^{\prime}, \ldots, \Gamma_{n}^{\prime}$ as in Theorem 5.1, we can compute a name of $N_{D}$.

\section{THE SLIT DISK DOMAIN}

We now wish to combine the previous results on analytic extension and Neuman problems with the constructions of M. Schiffer to demonstrate the computability of conformal mapping onto canonical slit domains. However, these constructions presume the input domain is a smooth Jordan domain. So that we may demonstrate these results for all finitely connected non-degenerate domains, we first prove the following.

Lemma 6.1 (Conformal Reconfiguring Lemma). Given a name of a finitely connected domain $D$, a name of its boundary, and the number of its boundary components, it is possible to compute, uniformly in these data, the following.

(1) A name of a conformal map $f$ of $D$ onto an unbounded analytic domain $D^{\prime}$ as well as names of analytic parameterizations of the boundary curves of $D^{\prime}$.

(2) A name of a conformal map $f$ of $D$ onto a bounded analytic domain $D^{\prime \prime}$ as well as names of analytic parameterizations of the boundary curves of $D^{\prime \prime}$. We can additionally ensure that the outermost boundary curve of $D^{\prime \prime}$ is a circle, $C$. If we are additionally given a name of a boundary component of $D, \gamma$, then we can ensure $\gamma$ corresponds to $C$ under $f$.

Finally, if $D$ is a smooth Jordan domain, and if we are additionally provided with names of the boundary curves of $D$ as well as their derivatives, then we can uniformly in all these data compute the homeomorphic extension of the maps in (1) and (2) to $\bar{D}$. 
Proof. We inductively define a sequence of domains and canonical maps as follows. We first compute distinct $\zeta_{0}, \zeta_{1} \in D$. Let

$$
f_{0}(z)=\frac{z-\zeta_{0}}{z-\zeta_{1}}
$$

Let $D_{0}=f_{0}[D]$. Hence, $0, \infty \in D_{0}$. Let $D_{0,1}, \ldots, D_{0, n}$ be the connected components of the complement of $D_{0}$. Let $f_{1}$ be the conformal map of $\hat{\mathbb{C}}-D_{0,1}$ onto the complement of a closed disk such that $f_{1}(\infty)=\infty, f_{1}^{\prime}(\infty)=1$, and $f_{1}(0)=0$. Let $D_{1}=f_{1}\left[D_{0}\right]$. Let $D_{1, j}=f_{1}\left[D_{0, j}\right]$ for $j=2, \ldots, n$.

Suppose $k \in \mathbb{N}$ and $f_{k}, D_{k}$, and $D_{k, 1}, \ldots, D_{k, n}$ have been defined. Let $k_{0} \in$ $\{1, \ldots, n\}$ be congruent to $k$ modulo $n$. Define $f_{k+1}$ to be the conformal map of the complement of $D_{k, k_{0}}$ onto the complement of a closed disk such that $f_{k+1}(\infty)=\infty$, $f_{k+1}^{\prime}(\infty)=1$, and $f_{k+1}(0)=0$. Let $D_{k+1, k_{0}}$ denote this disk. Let $D_{k+1}=f_{k+1}\left[D_{k}\right]$. Let $D_{k+1, j}=f_{k+1}\left[D_{k, j}\right]$ when $j \neq k_{0}$.

The construction we have just described is known as the Koebe construction. By Theorem 4.6 of [2], the sequences $\left\{f_{k}\right\}_{k},\left\{D_{k}\right\}_{k}$, and $\left\{\partial D_{k, j}\right\}_{k}$ can be computed uniformly from the given data. If we take $D^{\prime}=D_{n}$, we obtain all the information required by (1). By applying an inversion, we obtain (2). If $D$ is a smooth Jordan domain, and if we are additionally given names of the boundary curves of $D$ and their derivatives, the it follows from the Weak Computable Carathéodory Theorem (Theorem 3.2) that we can compute homeomorphic extensions of $f_{0}, \ldots, f_{n}$ and hence that of $g$.

Theorem 6.2 (Computability of slit disk mapping). Given a name of a finitely connected, non-degenerate domain D, a name of its boundary, a name of one of its boundary components, $\gamma$, a name of a point $\zeta_{0} \in \mathbb{D}$, and the number of boundary components of $D$, we can compute a conformal mapping of $D$ onto a slit disk domain that maps $\zeta_{0}$ to 0 and $\gamma$ to $\partial \mathbb{D}$. If $D$ is a smooth Jordan domain, and if we are also supplied with names of its boundary curves and their derivatives, then we can compute the continuous extension of this map to the closure of D.

Proof. We first apply the Conformal Reconfiguring Lemma. Let $f, D_{1}, \gamma_{1}, \ldots, \gamma_{n}$ be thusly obtained. We may assume $f$ maps $\gamma$ onto $\gamma_{n}$. Let $\zeta_{1}=f\left(\zeta_{0}\right)$. We can now compute the center and radius of $\gamma_{n}$. Label these $\xi$ and $R$ respectively. Let $D_{2}, \Gamma_{1}, \ldots, \Gamma_{n}, \zeta_{2}$ be the images of $D_{1}, \gamma_{1}, \ldots, \gamma_{n}, \zeta_{1}$ under the inversion map $z \mapsto \frac{R^{2}}{z-\xi}$.

It suffices to show that we can compute a conformal map $g$ of $D_{2}$ onto a slit disk domain such that $g\left(\zeta_{2}\right)=0$ and $g$ maps $\Gamma_{n}$ to $\partial \mathbb{D}$. We first define what this map is, and then show that it can be computed from the given data. The latter is facilitated by minor rewriting of the standard construction of $g$ due to M. Schiffer [17]. First, let $u$ be the continuous function on $\overline{D_{2}}$ such that $u$ is harmonic on $D_{2}$ and $u(\zeta)=-\log \left|\zeta-\zeta_{2}\right|$ for all $\zeta$ on the boundary of $D_{2}$. That is, $u$ is the Green's function of $D_{2}$ at $\zeta_{2}$. By Theorem 3.3, $u$ can be computed from the given data. Then, by Lemma 4.1, we can compute an analytic extension of $u$ on $D_{2}, \hat{u}$. Let $g(z)=\exp (\hat{u}(z))\left(z-\zeta_{2}\right)$. It follows that $g$ can be computed from the given data.

It is demonstrated in [17] (see equation (A1.21)) that $g$ is the conformal mapping of $D_{2}$ onto a slit disk domain that maps $\zeta_{2}$ to 0 and $\Gamma_{n}$ to $\partial \mathbb{D}$. For the sake of completeness, we will include a proof of this fact since it is not difficult. However, in order to stay with the current thread of computability, we delay doing so until the end of this proof. 
Now, suppose $D$ is a smooth Jordan domain and that we are given also names of the derivatives of its boundary curves. It then follows from Theorem 3.2 that we can compute the homeomorphic extension of $f$ to $\bar{D}$. Hence we can compute a homeomorphism $f_{1}$ of $\bar{D}$ onto $\overline{D_{2}}$ that is conformal on $D$. Hence, it suffices to show we can compute the continuous extension of $g$ to $\overline{D_{2}}$. To this end, it suffices to show that we can compute the continuous extension of $\hat{u}$ to $\overline{D_{2}}$. Let $v=\operatorname{Im}(\hat{u})$. Then, for all $z \in D_{2}$,

$$
v(z)=\int_{\zeta_{2}}^{z} \frac{\partial \operatorname{Re}(\hat{u})}{\partial n} d s .
$$

Hence, it suffices to compute a harmonic extension of the real part of $\hat{u}$ to a neighborhood of $\overline{D_{2}}$. To this end, let us first compute a rational number $r>0$ such that $\overline{D_{r}\left(\zeta_{2}\right)}$ is contained in $D_{2}$. Let $D_{2}^{\prime}=D_{2}-\overline{D_{r}\left(\zeta_{2}\right)}$, and let $\hat{u}_{2}(z)=\hat{u}(z)+\log \left(z-\zeta_{2}\right)$ for all $z \in D_{2}^{\prime}$. It follows that $\hat{u_{2}}$ is analytic in $D_{2}^{\prime}$ and has constant real part on every boundary component of $D_{2}^{\prime}$ except $\partial D_{r}\left(\zeta_{2}\right)$. It now follows from Theorem 3.4 that we can compute an open set $U$ that contains $\partial D_{2}$ and a harmonic extension of the real part of $\hat{u}_{2}$ to $D_{2}^{\prime} \cup U$. It now follows that we can compute a harmonic extension of the real part of $\hat{u}$ to a neighborhood of $D_{2}$.

We conclude by giving a short demonstration that $g$ is in fact the required conformal map. We first note that the modulus of $g$ is constant on each boundary curve. This follows from the construction of $g$. Let $r_{j}$ be the value of the modulus of $g$ on $\Gamma_{j}$. It follows from the construction of $g$ that $r_{n}=1$.

We now make some observations about the argument of $g$ on these boundary curves. By definition,

$$
\arg (g(\zeta))=\operatorname{Im}(\hat{u}(\zeta))+\arg \left(\zeta-\zeta_{2}\right) .
$$

Clearly, $\operatorname{Im}(\hat{u})$ has no net change as $\zeta$ travels around any boundary curve. As $\zeta$ travels around any of $\Gamma_{1}, \ldots, \Gamma_{n-1}, \arg \left(\zeta-\zeta_{2}\right)$ has no net change either. Hence, we can conclude $g$ maps each of $\Gamma_{1}, \ldots, \Gamma_{n-1}$ into a corresponding circular arc with center 0 which does not form an entire circle. Let $\sigma_{1}, \ldots, \sigma_{k-1}$ denote these arcs.

We now demonstrate that no point of modulus greater than 1 is in the range of $g$. For, if $w$ is such a point, then by what has just been shown, the argument of $g(\zeta)-w$ has no net change as $\zeta$ travels around any boundary curve of $D_{2}$. It then follows from the Argument Principle that $w$ is not in the range of $g$.

From this, we can now conclude that $\sigma_{1}, \ldots, \sigma_{n-1}$ all lie inside $\mathbb{D}$. So, let $\Omega=$ $\mathbb{D}-\left(\sigma_{1} \cup \ldots \cup \sigma_{n-1}\right)$. We naturally want to show that $g$ maps $D_{2}$ conformally onto $\Omega$. So, let $w \in \Omega$. Then, the argument of $g(\zeta)-w$ has a net change of $2 \pi$ as $\zeta$ travels around $\Gamma_{n}$, and no net change as $\zeta$ travels around any of $\Gamma_{1}, \ldots, \Gamma_{n-1}$. It follows from the Argument Principle that $w$ has exactly one preimage under $g$ in $D_{2}$. Hence, $g$ injectively maps $D_{2}$ onto $\Omega$. The injectivity of $g$ assures its conformality.

\section{Some immediate CONSEQUences of the Slit Disk Result}

Let 'SD' stand for 'slit disk', 'CS' for 'circular slit', etc.. We introduce some notation for the conformal maps onto these domains. Fix a non-degenerate, finitely connected domain $D$. Let $\zeta_{0}, \zeta_{1} \in D$, and let $\gamma_{1}, \ldots, \gamma_{n}$ be the boundary components of $D$. We then let $f_{S D}\left(\cdot ; D, \zeta_{0}, \gamma_{j}\right)$ denote the unique conformal map of $D$ onto a slit disk domain that maps $\zeta_{0}$ to 0 and $\gamma_{j}$ onto $\partial \mathbb{D}$ whose derivative at $\zeta_{0}$ is positive. 
Let $f_{C S}\left(\cdot ; D, \zeta_{0}, \zeta_{1}\right)$ be the conformal map of $D$ onto a circular slit domain that maps $\zeta_{0}$ to $0, \zeta_{1}$ to $\infty$, and whose residue at $\zeta_{1}$ is 1 .

Let $f_{P S}\left(\cdot ; D, \zeta_{1}, \theta\right)$ be the conformal map of $D$ onto a parallel slit domain where all slits have angle $\theta$ with the $x$-axis and whose Laurent expansion at $\zeta_{1}$ is of the form

$$
\frac{1}{z-\zeta_{1}}+a\left(z-\zeta_{1}\right)+b\left(z-\zeta_{1}\right)^{2}+\ldots
$$

Let $f_{R S}\left(\cdot ; D, \zeta_{0}, \zeta_{1}\right)$ be the conformal map of $D$ onto a radial slit domain that maps $\zeta_{0}$ to $0, \zeta_{1}$ to $\infty$, and whose residue at $\zeta_{1}$ is 1 .

Let $f_{S A}\left(\cdot ; D, \gamma_{j}, \gamma_{k}\right)$ be the conformal map of $D$ onto a slit annulus domain that maps $\gamma_{j}$ onto $\partial \mathbb{D}$ and $\gamma_{k}$ onto the inner circle.

We omit any of these parameters when they are made clear by context.

Suppose we are given a name of a finitely connected, non-degenerate domain $D$, a name of its boundary, and the number of its boundary components. It is now required to show that we can compute these other canonical maps uniformly in the parameters beyond the semicolon. In the case of $f_{C S}$ and $f_{S A}$, this follows from the following identities which are proven in [17].

$$
\begin{aligned}
f_{C S}\left(z ; \zeta_{0}, \zeta_{1}\right) & =\frac{f_{S D}^{\prime}\left(\zeta_{1} ; \zeta_{1}\right)}{f_{S D}^{\prime}\left(\zeta_{1} ; \zeta_{0}\right)} \frac{f_{S D}\left(z ; \zeta_{0}\right)}{f_{S D}\left(z ; \zeta_{1}\right)} \\
f_{S A}\left(z ; \gamma_{j}, \gamma_{k}\right) & =\frac{f_{S D}\left(z ; \zeta_{0}, \gamma_{j}\right)}{f_{S D}\left(z ; \zeta_{0}, \gamma_{k}\right)}
\end{aligned}
$$

It then follows that when $D$ is a smooth Jordan domain and we are in addition granted names of the boundary curves of $D$ and their derivatives, we can uniformly in all these data calculate the continuous extensions of $f_{C S}\left(\cdot ; \zeta_{0}, \zeta_{1}\right) f_{S A}\left(\cdot ; \gamma_{j}, \gamma_{k}\right)$ to $\bar{D}$.

We now discuss the computation of $f_{P S}(\cdot ; D, \zeta, \theta)$. Let $\zeta_{x}, \zeta_{y}$ denote the real and imaginary parts of $\zeta$ respectively. It is shown on pages 256 and 257 of [17] that

$$
\begin{aligned}
f_{P S}(z ; D, \zeta, \pi / 2) & =-\frac{\partial}{\partial \zeta_{x}} \log f_{S D}\left(z ; D, \zeta, \gamma_{n}\right) \\
f_{P S}(z ; D, \zeta, 0) & =-\frac{1}{i} \frac{\partial}{\partial \zeta_{y}} \log f_{S D}\left(z ; D, \zeta, \gamma_{n}\right) \\
f_{P S}(z ; D, \zeta, \theta) & =e^{i \theta}\left[\cos (\theta) f_{P S}(z ; D, \zeta, 0)-i \sin (\theta) f_{P S}(z ; D, \zeta, \pi / 2)\right] .
\end{aligned}
$$

The upshot of all this is that we can compute $f_{P S}(\cdot, D, \zeta, \theta)$ uniformly from the given data and $\theta$ if we can compute the derivative of the map $\log f_{S D}\left(z ; D, \cdot, \gamma_{n}\right)$. This requires some unwinding of the construction of $f_{S D}$. We refer back to the notation of the proof of Theorem 6.2. We first wish to more explicitly denote this construction as a function of $\zeta$. To this end, let us replace $b_{j}$ with $b_{j}(\zeta), u(z)$ with $u(z ; \zeta)$, and $\hat{u}(z)$ with $\hat{u}(z ; \zeta)$. Let $p_{j}(\zeta)$ denote the period of $u(\cdot ; \zeta)$ around $\Gamma_{j}$. It follows from the proof of Lemma 4.1 that

$$
\left(\begin{array}{c}
b_{1}(\zeta) \\
\cdot \\
\cdot \\
\cdot \\
b_{n-1}(\zeta)
\end{array}\right)=\left(P_{i, j}\right)_{i, j}^{-1}\left(\begin{array}{c}
-p_{1}(\zeta) \\
\cdot \\
\cdot \\
\cdot \\
-p_{n-1}(\zeta)
\end{array}\right)
$$


where $\left(P_{i, j}\right)_{i, j}$ is the Riemann matrix of $D_{2}$. The key point here is that $b_{j}(\zeta)$ can be expressed as a linear combination of $p_{1}(\zeta), \ldots, p_{n-1}(\zeta)$ and that the scalars in this combination are computable uniformly from the given data. Let $G$ denote the Green's function of $D_{2}$. As remarked in the proof of Theorem 6.2, $u(z ; \zeta)=G(z ; \zeta)+\log |z-\zeta|$. The period of $z \mapsto \log |z-\zeta|$ about any of $\Gamma_{1}, \ldots, \Gamma_{n-1}$ is zero. It follows that $p_{j}(\zeta)=\omega_{j}(\zeta)$, and we can certainly compute the derivative of $\omega_{j}$ ! So, uniformly in the given data and $z$, we can compute the derivative of $f_{S D}\left(z ; D_{2}, \cdot, \Gamma_{n}\right)$. However, $f_{S D}\left(\cdot ; D_{2}, \zeta, \Gamma_{n}\right)=f_{S D}\left(\cdot ; D, f^{-1}(\zeta), \gamma_{n}\right)$. The computability of $f_{S D}$ is thus resolved.

The only remaining task for this section is to show that if $D$ is a smooth Jordan domain and if we are additionally given names of the boundary curves of $D$ and their derivatives, then we can compute uniformly in all these data the continuous extension of $f_{P S}\left(\cdot ; D, \zeta, \gamma_{n}\right)$ to $\bar{D}$. This entails computing the derivative of $f_{S D}\left(\cdot ; D_{2}, \zeta, \Gamma_{n}\right)$ on $\overline{D_{2}}$. However, as has been noted, the computation of this map's derivative reduces to that of each $\omega_{j}$ (for $D_{2}$ ). Theorem 3.4 allows us to extend this to the closure of $D_{2}$.

\section{THE RADIAL SLIT DOMAIN}

We conclude with the following.

Theorem 8.1. From a name of non-degnerate, finitely connected, domain $D$, a name of its boundary, names of distinct $\zeta_{0}, \zeta_{1} \in D$, and the number of its boundary components, we can compute a name of $f_{R S}\left(\cdot ; D, \zeta_{0}, \zeta_{1}\right)$. If $D$ is a smooth Jordan domain, and if we are additionally given names of the boundary curves of $D$ and their derivatives, then we can uniformly in all these data compute the continuous extension of $f_{R S}\left(\cdot ; D, \zeta_{0}, \zeta_{1}\right)$ to $\bar{D}$.

Proof. By the Conformal Reconfiguring Lemma (Lemma 6.1), we can first compute a conformal map $f$ of $D$ onto a bounded analytic domain $D_{1}$. We can also compute analytic parameterizations of the boundary curves of $D_{1}$. Let $\Gamma_{1}, \ldots, \Gamma_{n}$ be these boundary curves. Let $\zeta_{0}^{\prime}=f\left(\zeta_{0}\right)$, and let $\zeta_{1}^{\prime}=f\left(\zeta_{1}\right)$.

We use another of M. Schiffer's constructions; namely, the main idea of the proof of ??? of [17]. Again, we rewrite things a little in order to make the computability more transparent.

Let $u_{1}(z)=N_{D_{1}}\left(z, \zeta_{0}^{\prime}\right)-N_{D_{1}}\left(z, \zeta_{1}^{\prime}\right)+\log \left|z-\zeta_{0}^{\prime}\right|-\log \left|z-\zeta_{1}^{\prime}\right|$. Hence, $u_{1}$ is harmonic in $D_{1}$. We can compute a name of $u_{1}$ uniformly from the given data. In addition,

$$
\int_{\Gamma_{j}} \frac{\partial u_{1}}{\partial n} d s=-\frac{2 \pi l}{L}+\frac{2 \pi l}{L}+0-0=0
$$

where $l$ is the length of $\Gamma_{j}$. Hence, $u_{1}$ has a single-valued harmonic conjugate, $v_{1}$. We can also compute $v_{1}$ from the given data. Let $f_{1}=u_{1}+i v_{1}$, and let

$$
g(z)=\exp \left(f_{1}(z)\right) \frac{z-\zeta_{1}^{\prime}}{z-\zeta_{0}^{\prime}}
$$

Again, it is shown in the proof of (A1.62) of [17] that $g$ is the required conformal map of $D_{1}$ onto a radial slit domain. But, the proof is short and so we include it 
here. Let $\zeta, z \in \Gamma_{j}$. Then,

$$
\begin{aligned}
v_{1}(z)-v_{1}(\zeta) & =\int_{\zeta}^{z} \frac{\partial v_{1}}{\partial s} d s \\
& =\int_{\zeta}^{z} \frac{\partial u_{1}}{\partial n} d s \\
& =\arg \left(z-\zeta_{0}^{\prime}\right)-\arg \left(z-\zeta_{1}^{\prime}\right)-\arg \left(\zeta-\zeta_{0}^{\prime}\right)+\arg \left(\zeta-\zeta_{1}^{\prime}\right)
\end{aligned}
$$

It follows that $\arg (g)$ is constant on each $\Gamma_{j}$.

To finish the proof, it suffices to show we can compute the continuous extension of $g$ to $\overline{D_{1}}$. To this end, it suffices to show we can compute from the given data the continuous extension of $v_{1}$ to the closure of $D_{1}$. However, if we examine the process of solving the Neumann problem, we see that it is $v_{1}$ that is computed first and that $u_{1}$ is computed as a result. Futhermore, $v_{1}$ is obtained as a solution of a Dirichlet problem. Hence, we automatically have computed $\arg (g)$ on $\overline{D_{1}}$. However, $\arg (g)$ is constant on each $\Gamma_{j}$, and we can compute these constants. Let $\alpha_{j}$ denote the value of $\arg (g)$ on $\Gamma_{j}$. Hence,

$$
v_{1}(z)=\sum_{j=1}^{n} \alpha_{j} \omega\left(z, \Gamma_{j}, D_{1}\right)-\arg \left(\frac{z-\zeta_{1}^{\prime}}{z-\zeta_{0}^{\prime}}\right) .
$$

We then compute $r$ such that $\overline{D_{r}\left(\zeta_{0}^{\prime}\right)} \subseteq D_{1}$. (Actually, this is part of the process of computing a name of $\zeta_{0}^{\prime}$.) Then,we can compute an open set $U$ containing $\Gamma_{1}, \ldots, \Gamma_{n}$ and an extension of $v_{1}$ to $D_{1} \cup U$. We can then use the Cauchy-Riemann equations to compute an extension of $u_{1}$ to $D_{1} \cup U$.

\section{ACKNOWLEDGEMENTS}

The second author thanks his wife Susan for her support.

\section{REFERENCES}

1. N. I. Akhiezer, Aerodynamical investigations, vol. 7, Ukrain. Akad. Nauk Trudi Fiz.-Mat. Viddilu, 1928.

2. V.V. Andreev, D. Daniel, and T. H. McNicholl, Computing conformal maps onto circular domains, Submitted. Preprint avaliable at http://www.cs.lamar.edu/faculty/mcnicholl/index.php.

3. E. Bishop and D.S. Bridges, Constructive analysis, Springer (Berlin), 1985.

4. J.B. Conway, Functions of one complex variable i, 2nd ed., Graduate Texts in Mathematics, vol. 11, Springer-Verlag, 1978.

5. D. Crowdy, Schwarz-christoffel mappings to unbounded multiply connected polygonal regions, Mathematical Proceedings of the Cambridge Philosophical Society 142 (2007), no. 2, 319-339.

6. T. K. Delillo, Schwarz-christoffel mapping of bounded multiply connected domains, Computational Methods Function Theory 6 (2006), 275-300.

7. D. Gaier, Untersuchung zur durchführung der konformen abbildung mehrfach zusammenhängender gebiete, Arch. Rat. Mech. Anal. 3 (1959), 149 - 178.

8. J.B. Garnett and D. E. Marshall, Harmonic measure, New Mathematical Monographs, 2, Cambridge University Press, Cambridge, 2005.

9. N. D. Halsey, Potential flow analysis of multielement airfoils using conformal mapping, American Institute of Aeronautics and Astronautics. Journal 17 (1979), no. 12, 1281-1288.

10. P. Henrici, Applied and computational complex analysis. vol. 3., Pure and Applied Mathematics, Wiley \& Sons, Inc., New York, 1986.

11. P. Hertling, An effective Riemann Mapping Theorem, Theoretical Computer Science 219 (1999), $225-265$ 
12. P. Koebe, Über die konforme Abbildung mehrfach-zusammenhängender Bereiche, Jahresber. Dt. Math. Ver. 19 (1910), 339 - 348.

13. _ Abhandlungen zur theorie der konformen abbildung, iv, Acta Math 41 (1918), 305344.

14. V. V. Mityushev and S. V. Rogosin, Constructive methods for linear and nonlinear boundary value problems for analytic functions, CRC Monographs and Surveys in Pure and Applied Mathematics, vol. 108, Chapman and Hall, 2000.

15. Z. Nehari, Conformal mapping, Dover, 1975.

16. M. B. Pour-El and I. J. Richards, Computability in analysis and physics, Perspectives in Mathematical Logic, Spring-Verlag, Berlin, 1989.

17. M. Schiffer, Some recent developments in the theory of conformal mapping, Appendix to: R. Courant. Dirichlet Principle, Conformal Mapping and Minimal Surfaces, Interscience Publishers, 1950.

18. K. Weihrauch, Computable analysis, an introduction, Springer-Verlag, 2000.

19. K. Weihrauch and T. Grubba, Elementary computable topology, Journal of Universal Computer Science 15 (2009), no. 6, 1381-1422.

20. M. Ziegler and V. Brattka, Computability in linear algebra, Theoretical Computer Science 326 (2004), 187-211.

Department of Mathematics, Lamar University, Beaumont, Texas 77710

E-mail address: andreev@math.lamar.edu

Department of Mathematics, Lamar University, Beaumont, Texas 77710

E-mail address: timothy.h.mcnicholl@gmail.com 